UDC 621.397.2

SCOPUS CODE 1711

https://doi.org/10.36073/1512-0996-2020-4-68-74

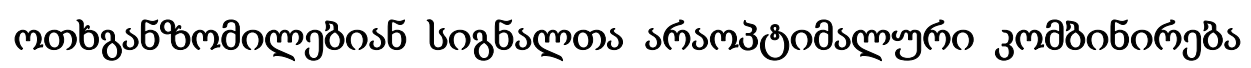

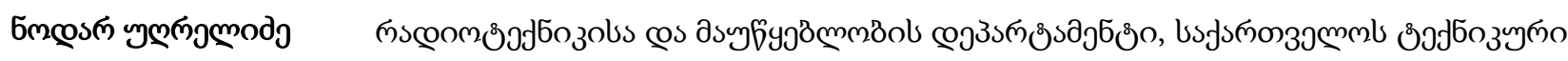

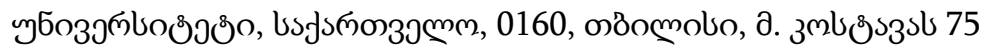

E-mail: toban555@gmail.com

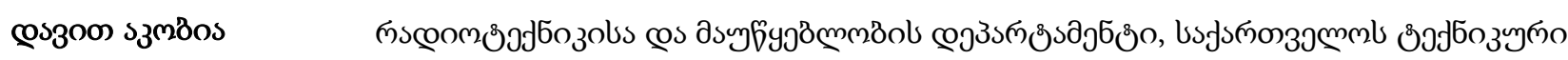

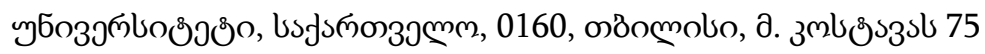

E-mail: dato41814@gmail.com

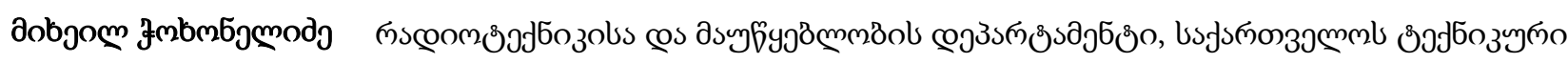

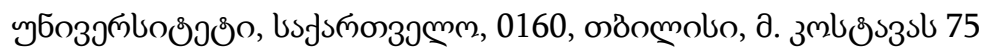

E-mail: misha.chokhon@gmail.com

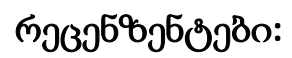

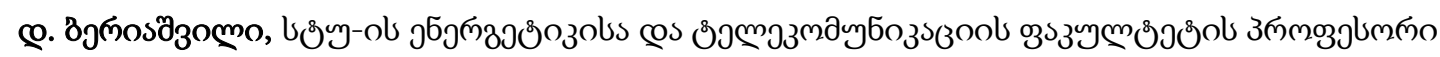

E-mail: d.beriashvili@gtu.ge

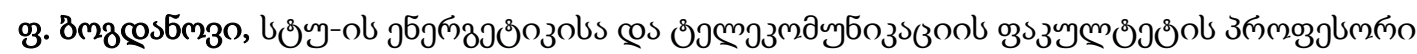

E-mail: faik.bogdanov@emcos.com

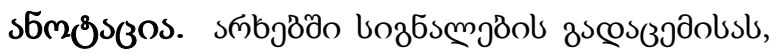

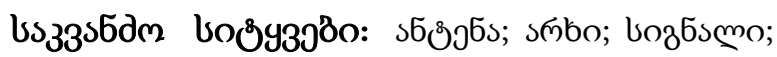

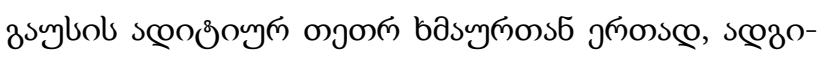

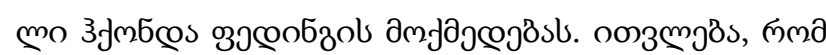

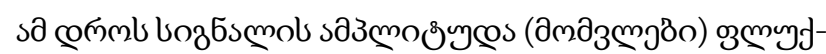

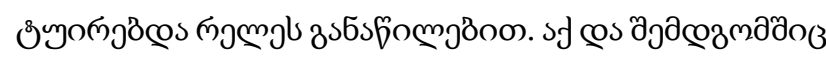

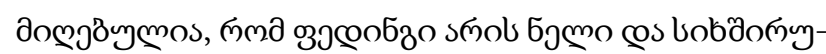
мsழ sলিslym

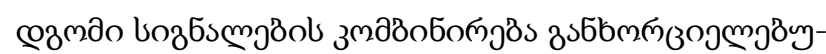

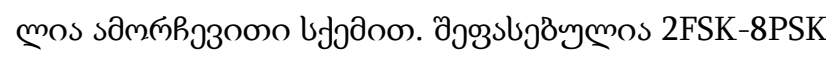

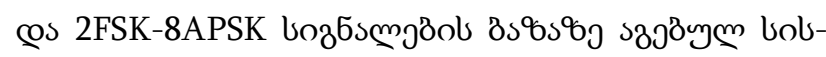

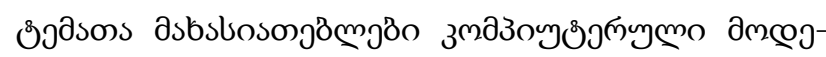

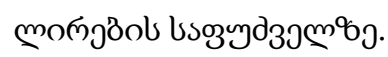
зэщебар.

\section{gjlsзšmo}

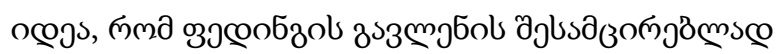

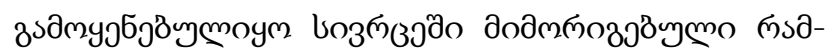

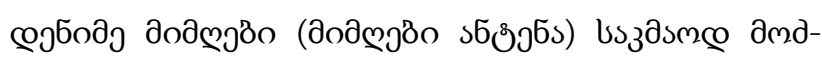

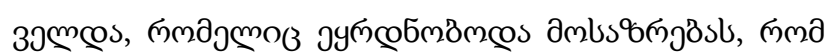

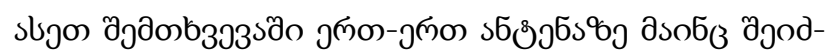

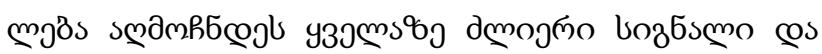

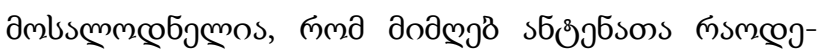

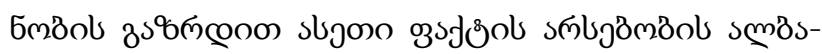

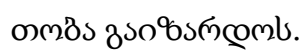




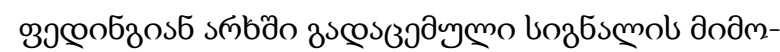

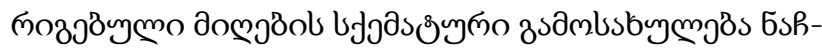

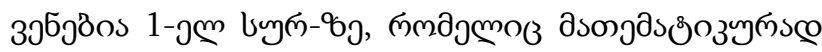

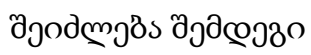

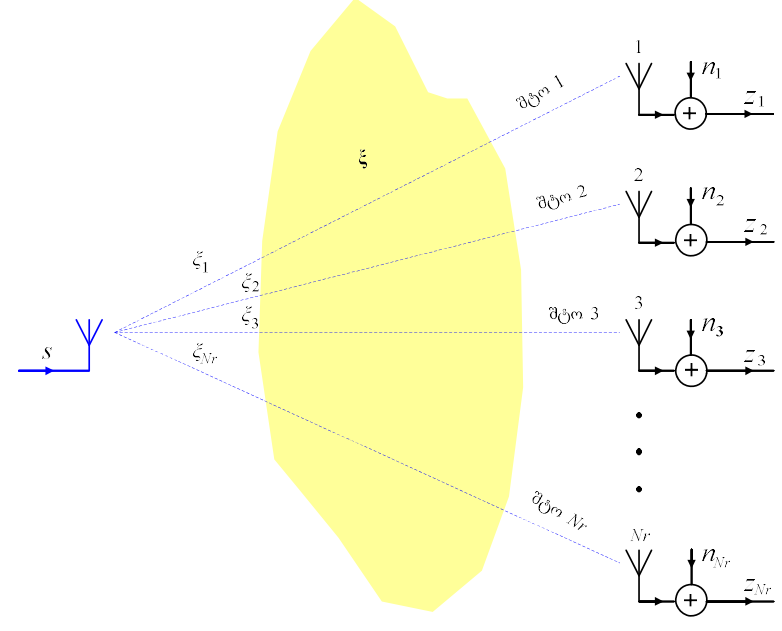

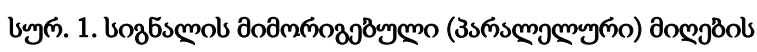
зиобъозулmo bjјаs

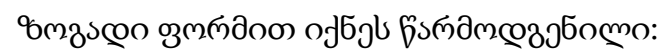

$$
\mathbf{z}=\xi \mathbf{s}+\mathbf{n},
$$

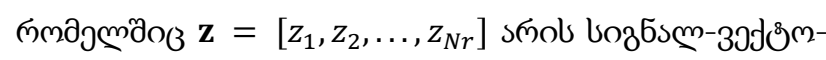

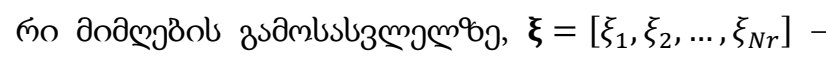

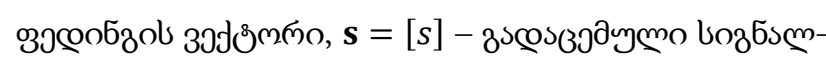

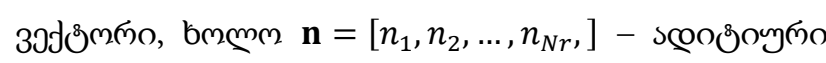

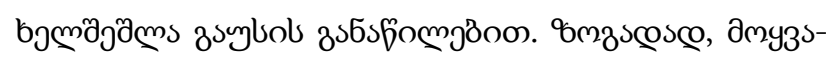

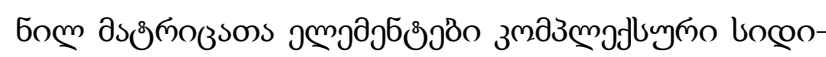

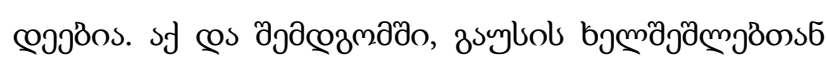

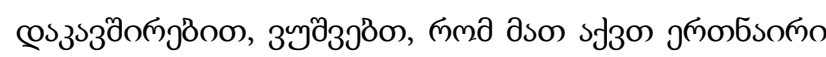

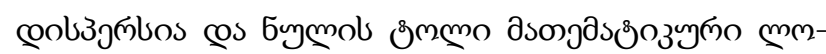
co6o.

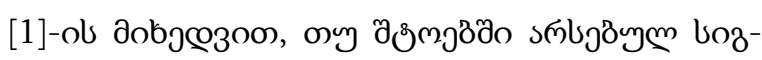

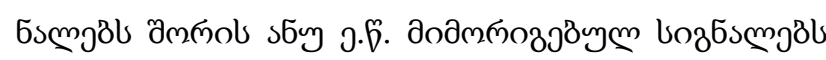

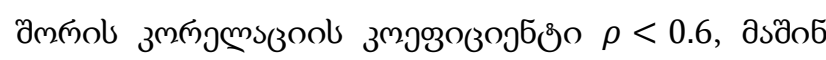

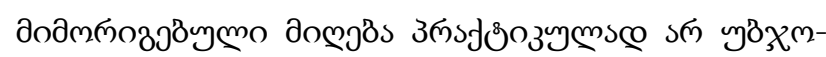

ISSN 1512-0996

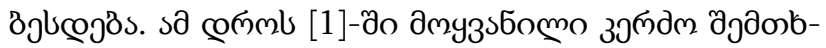
393obsonzol hsбl (ob. [1], ๒у⿰п. 5. 1), пмд додмэठ

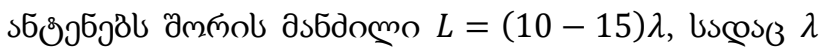

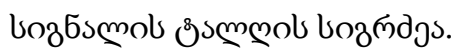

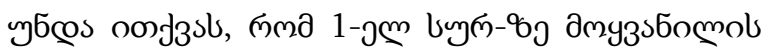

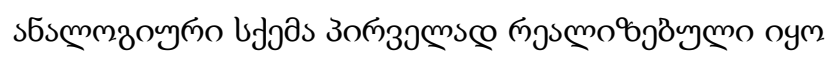

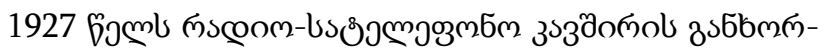

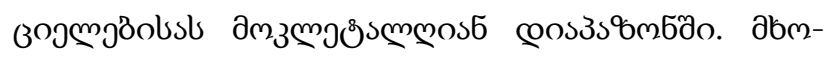

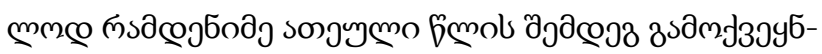

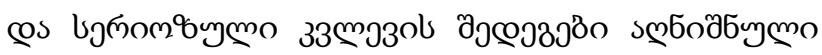

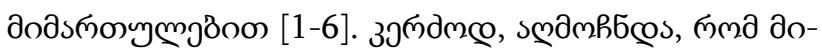

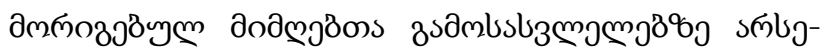

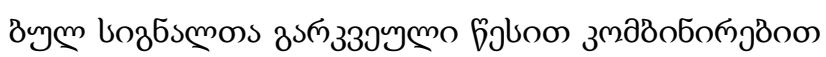

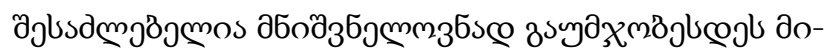

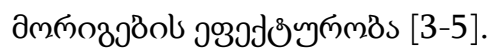

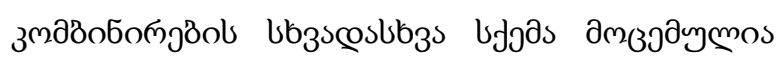

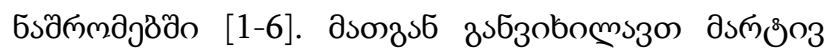

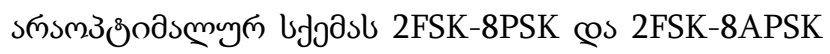

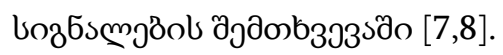

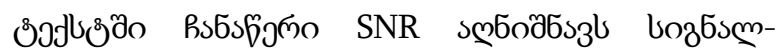

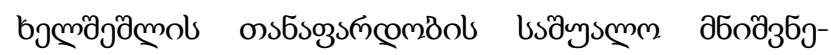

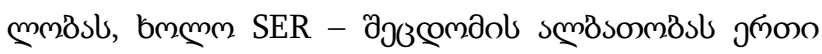

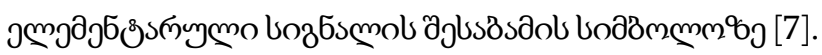

\section{domooscen 5sfomo}

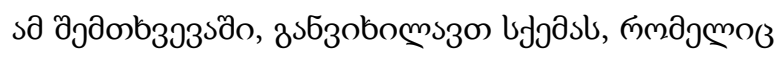

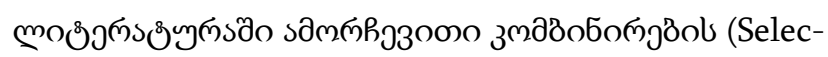

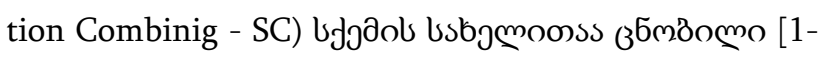

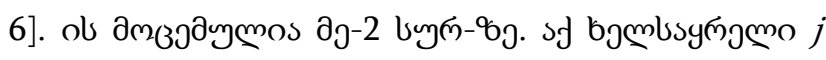

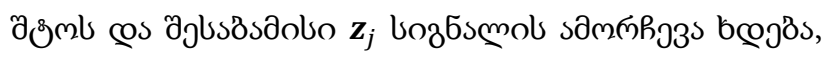

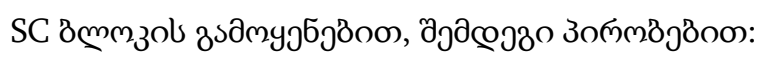

$$
z_{j}=\max _{\varsigma}\left[\mathrm{W} \mathbf{z}_{\varsigma}\right], \varsigma \in\left\{1,2, \ldots, N_{r}\right\},
$$

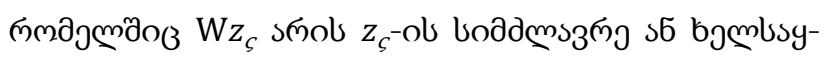




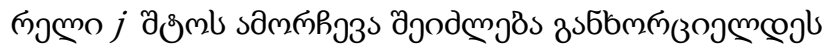

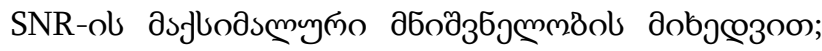

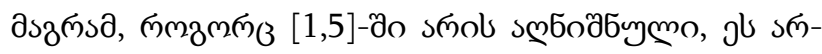

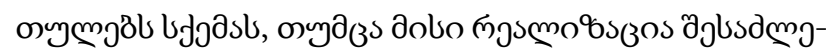

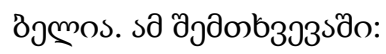

$$
z_{j}=\max _{\varsigma}\left[\operatorname{SNR}_{\varsigma}\right], \varsigma \in\left\{1,2, \ldots, N_{r}\right\} .
$$

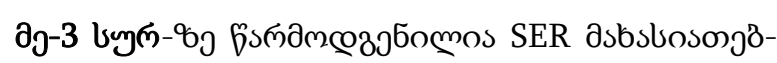

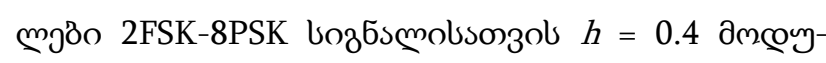

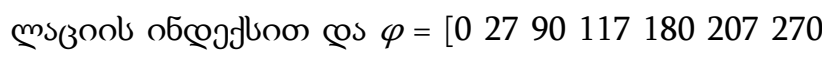

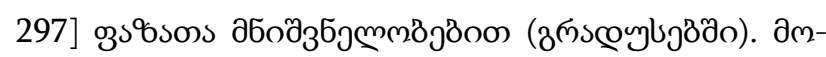

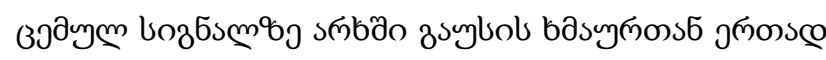

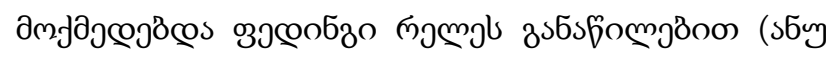

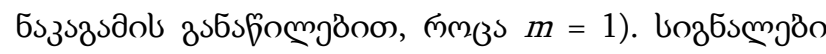
доощృ

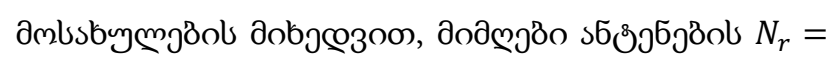

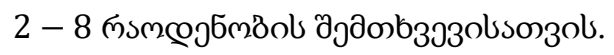

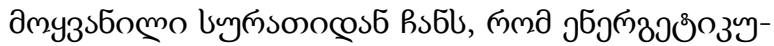

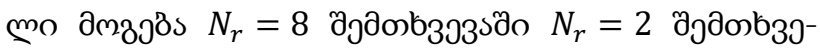

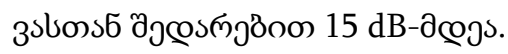

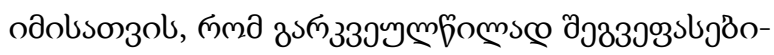

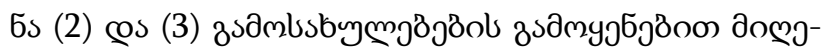

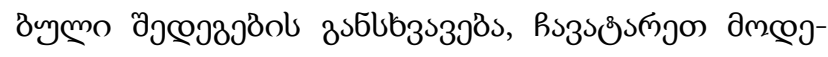

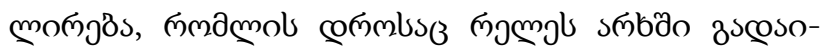
зəдм@s 2FSK-8PSK ( $h=0.4)$ œo 2FSK-8APSK $(h=0.3)$

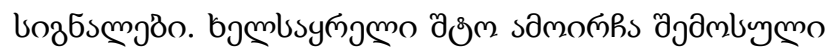

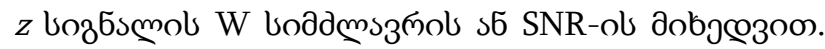

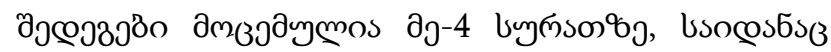

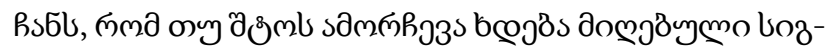
Бsmol boddmsз̆

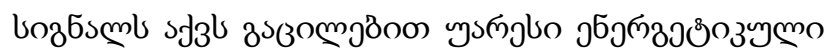

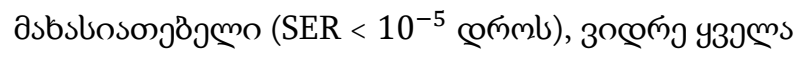

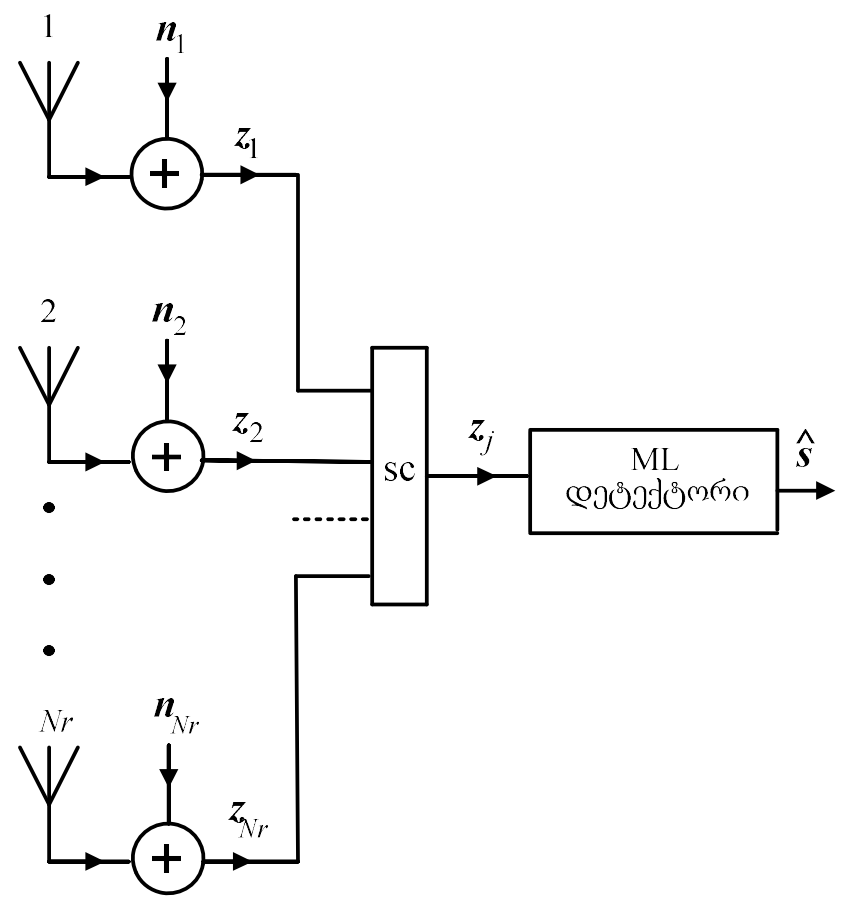

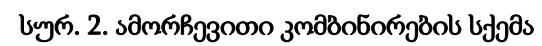




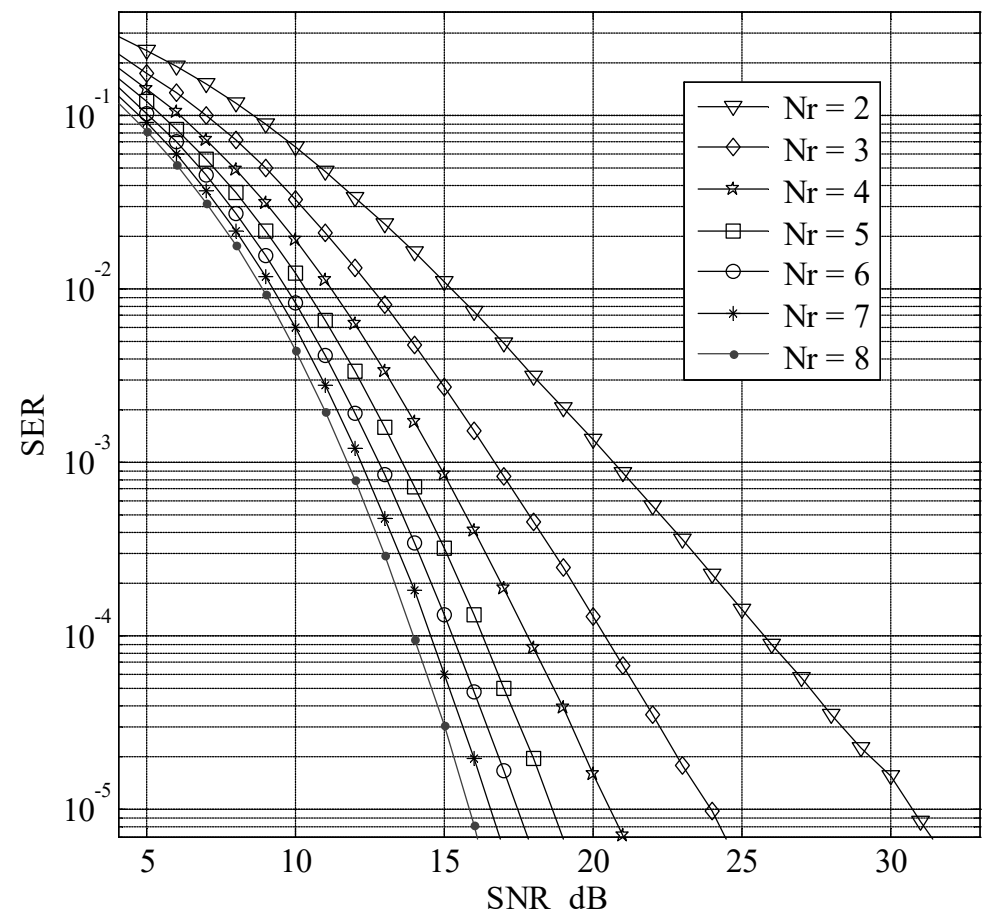

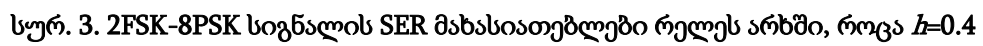

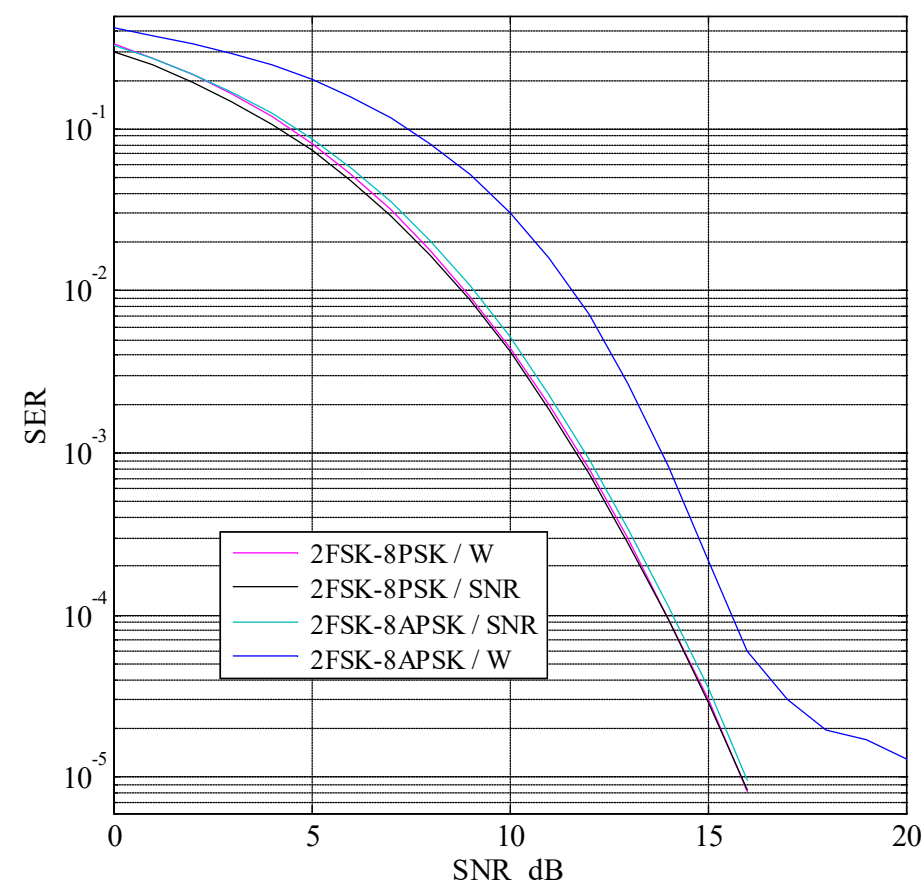

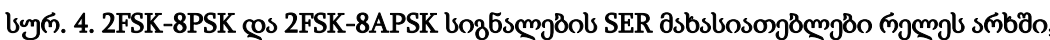

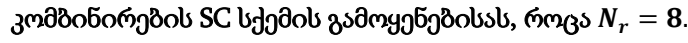




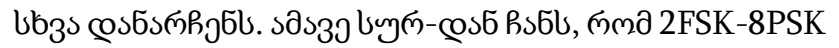

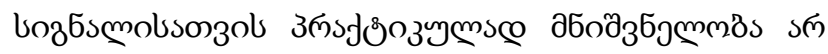

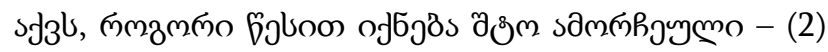

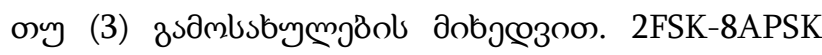

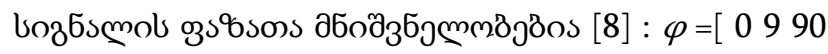
99180189270 279].

\section{$\cos 33^{6 s}$}

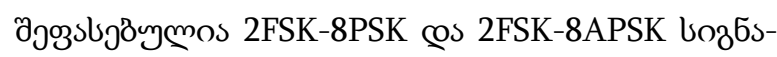

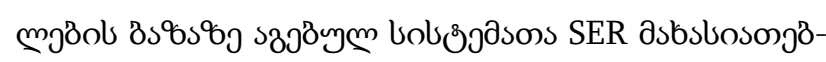

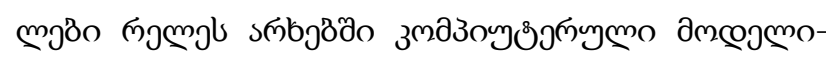

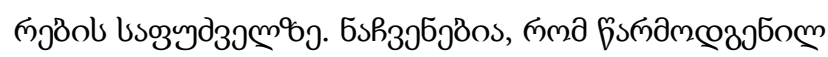

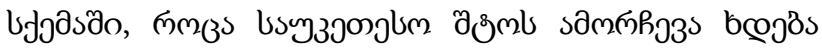

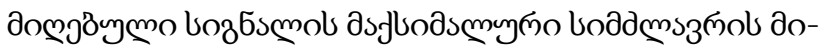

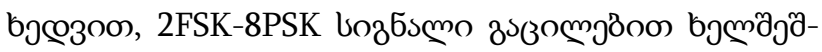

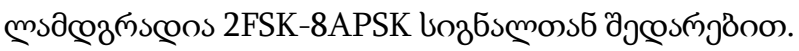

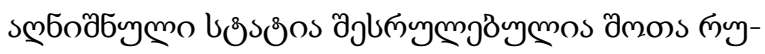

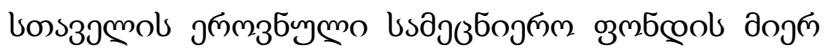

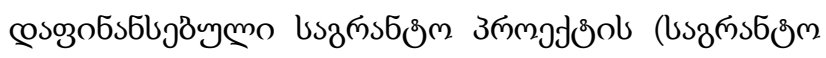

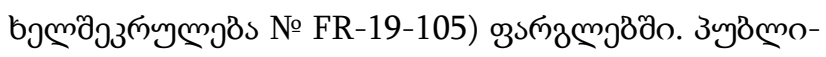

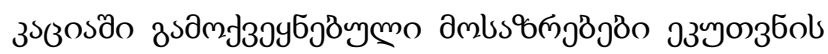

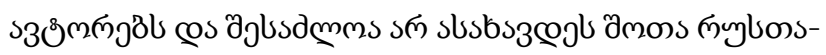

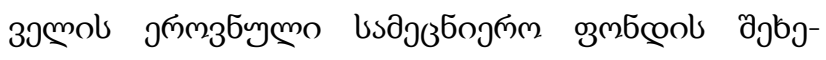

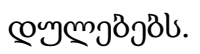

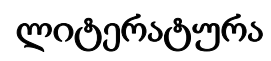

1. Zyuko A. G. Efficiency and noise immunity of communication systems. M.: "Sviaz", 1972. (In Russian).

2. Sklar B. Digital communications. 2th ed. by Prentice Hall PTR. 2001.

3. Brennan D.G. Linear diversity combining technique. Proceeding of the IRE. Vol. 47. 1959, 1075-1102 pp.

4. Isomäki P., Isoaho J. On diversity combining. University of Turku. Turku Centre for Computer Science. Technical report. 2008, 1-25 pp.

5. Fink L.M. The theory of discrete messages transmission system. M.: „Sov. Radio”. 1970. (In Russian).

6. Lee W.C.Y. Mobile communications engineering. Publisher: "McGraw-Hill Professional". 1st Edition. 1982.

7. Ugrelidze N., Shavgulidze S., Sordia M. New four-dimensional signal constellations construction. IET communications journal. Vol. 14. Iss. 10. 2020, 1554-1559 pp.

8. Ugrelidze N., Shavgulidze S. Combined frequency and amplitude-phase shift keying modulation in a fourdimensional space. International journal of electronics and communications (AEÜ), submitted for publication. 
UDC 621.396.946

SCOPUS CODE 1711

https://doi.org/10.36073/1512-0996-2020-4-68-74

\title{
Sub-optimal combining of four-dimensional signals
}

$\begin{array}{ll}\text { Nodar Ugrelidze } & \text { Department of Radio-Engineering and Broadcasting, Georgian Technical University, } 75 \\ & \text { M. Kostava str, 0160 Tbilisi, Georgia } \\ & \text { E-mail: toban555@gmail.com } \\ \text { Department of Radio-Engineering and Broadcasting, Georgian Technical University, } 75 \\ \text { M. Kostava str, 0160 Tbilisi, Georgia } \\ \text { E-mail: dato41814@gmail.com } \\ \text { Department of Radio-Engineering and Broadcasting, Georgian Technical University, } 75 \\ \text { Mikheil } \\ \text { Chokhonelidze }\end{array}$

\section{Reviewers:}

D. Beriashvili, Professor, Faculty of Power Engineering and Telecommunication, GTU

E-mail: d.beriashvili@gtu.ge

F. Bogdanov, Professor, Faculty of Power Engineering and Telecommunication, GTU

E-mail: faik.bogdanov@emcos.com

\begin{abstract}
The article discusses the issue of signal transmission in channels where fading takes place along with additive white Gaussian noise. It is commonly supposed that at this time the signal amplitude (envelope) was fluctuated with the Rayleigh distribution. It is accepted that fading is slow and frequency is non-selective. For transmit diversity from antennas, their combination with signals is carried out in a selective scheme. The characteristics of systems based on 2FSK-8PSK and 2FSK-8APSK signals are evaluated with the help of computer simulation.
\end{abstract}

Key words: Antenna; channel; fading; signal. 
UDC 621.396.946

SCOPUS CODE 1711

https://doi.org/10.36073/1512-0996-2020-4-68-74

\title{
Суб-оптимальное комбинирование четырехмерных сигналов
}

Нодар Угрелидзе Департамент радиотехники и радиовещания, Грузинский технический университет, Грузия, 0160, Тбилиси, ул. М. Костава, 75

E-mail: toban555@gmail.com

Давид Акобия Департамент радиотехники и радиовещания, Грузинский технический университет, Грузия, 0160, Тбилиси, ул. М. Костава, 75

E-mail: dato41814@gmail.com

Михаил Чохонелидзе Департамент радиотехники и радиовещания, Грузинский технический университет, Грузия, 0160, Тбилиси, ул. М. Костава, 75

E-mail: misha.chokhon@gmail.com

\section{Рецензенты:}

Д. Бериашвили, профессор факультета энергетики и телекоммуникаций ГТУ

E-mail: d.beriashvili@gtu.ge

$\Phi$. Богданов, профессор факультета энергетики и телекоммуникаций ГТУ

E-mail: faik.bogdanov@emcos.com

Аннотация. Фединг происходил с гауссовым аддитивным белым шумом при передаче сигналов по каналам. Считается, что в это время амплитуда (огибающая) сигнала имеет распределение Рэлея. Принято здесь и в дальнейшем, что фединг происходит медленно и частотно не избирательно. Дальнейшее комбинирование сигналов, разнесённых антенн, осуществляется схемой выбора. Оценены характеристики систем на основе сигналов 2FSK-8PSK и 2FSK-8APSK с помощью компьютерного моделирования.

Ключевые слова: антенна; канал; сигнал; фединг.

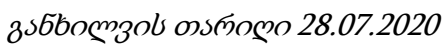

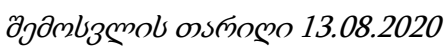

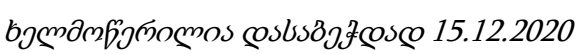

